\title{
Uso da simulação realística na segurança da equipe cirúrgica frente ao coronavírus: relato de experiência
}

\author{
Use of realistic simulation in the safety of the surgical team against coronavirus: experience report \\ Uso de simulación realista en seguridad del equipo quirúrgico ante coronavirus: informe de
} experiencia

Recebido: 0/08/2021 | Revisado: 09/08/2021 | Aceito: 12/08/2021 | Publicado: 16/08/2021

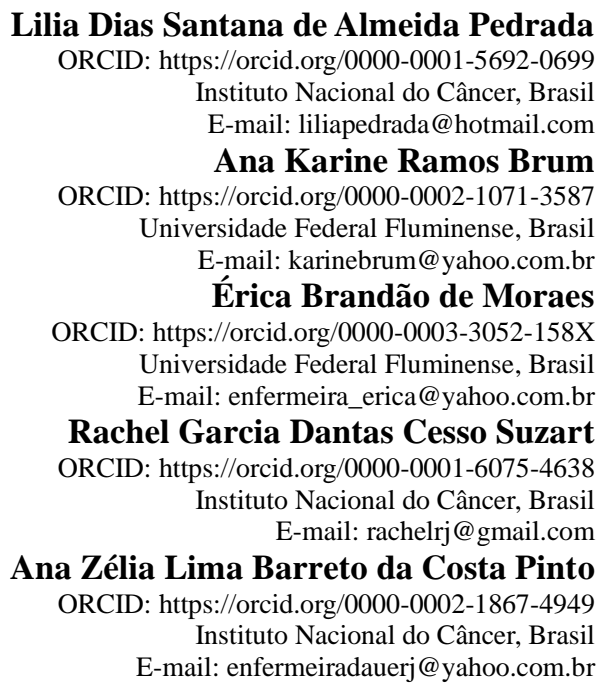

\begin{abstract}
Resumo
O objetivo deste relato de experiência é descrever a prática e os resultados do uso da simulação realística in situ, a fim de simular o fluxo e atendimento do paciente cirúrgico com suspeita ou confirmação de contaminação do SARS-CoV2 no período transoperatório. Participaram desse treinamento enfermeiros; técnicos de enfermagem; anestesistas; residentes de anestesia e enfermagem; maqueiros e profissionais de higienização. Utilizou-se a estratégia de simulação de alto rendimento através da Prática Deliberada de Ciclos Rápidos (PDCR). A memorização de cada participante foi repetida inúmeras vezes, a fim de reduzir o erro e objetivando atingir a maestria. Ao final do treinamento foi realizado o debriefing para extrair contribuições dos participantes e reforçar os pontos positivos, não valorizando o erro, mas mostrando a solução. Foi importante compreender os sentimentos de ansiedade e medo que os profissionais envolvidos tinham sobre o assunto, e esse fator colaborou e influenciou para a estratégia metodológica. Percebeu-se que todos os profissionais da linha de frente da cirurgia, que estavam envolvidos no processo da prática educativa de simulação realística, sentiram-se mais seguros e confiantes para o enfrentamento da cirurgia de emergência de pacientes suspeitos ou contaminados pelo novo coronavírus.
\end{abstract}

Palavras-chave: Pessoal de saúde; Simulação; Segurança do paciente; Infecções por coronavírus.

\begin{abstract}
The purpose of this experience report is to describe the practice and results of using realistic simulation in situ, in order to simulate the flow and care of the surgical patient with suspected or confirmed contamination of SARS-CoV-2 in the transoperative period. Nurses participated in this training; nursing technicians; anesthetists; anesthesia and nursing residents; makeup artists and hygiene professionals. The high performance simulation strategy was used through the Deliberate Practice of Fast Cycles (PDCR). The memorization of each participant was repeated numerous times, in order to reduce the error and aiming to achieve mastery. At the end of the training, debriefing was carried out to extract contributions from the participants and reinforce the positive points, not valuing the error, but showing the solution. It was important to understand the feelings of anxiety and fear that the professionals involved had on the subject, and this factor collaborated and influenced the methodological strategy. It was noticed that all professionals in the front line of surgery, who were involved in the process of educational practice of realistic simulation, felt safer and more confident in coping with the emergency surgery of patients suspected of or infected with the new coronavirus.
\end{abstract}

Keywords: Health personnel; Simulation; Patient safety; Coronavirus infections. 


\begin{abstract}
Resumen
El propósito de este informe de experiencia es describir la práctica y los resultados del uso de simulación realista in situ, con el fin de simular el flujo y la atención de pacientes quirúrgicos con sospecha o confirmación de contaminación por SARS-CoV-2 en el período transoperatorio. Las enfermeras participaron en esta formación; técnicos de enfermería; anestesistas; residentes de anestesia y enfermería; camillas y profesionales sanitarios. Se utilizó la estrategia de simulación de alto rendimiento a través de la Práctica Deliberada de Ciclos Rápidos (PDCR). La memorización de cada participante se repitió innumerables veces, con el fin de reducir el error y con el objetivo de lograr el dominio. Al finalizar la formación se realizó un debriefing para extraer aportes de los participantes y reforzar los puntos positivos, no valorando el error, sino mostrando la solución. Era importante comprender los sentimientos de ansiedad y miedo que los profesionales involucrados tenían sobre el tema, y este factor colaboró e influyó en la estrategia metodológica. Se notó que todos los profesionales en primera línea de cirugía, que se involucraron en el proceso de práctica educativa de simulación realista, se sintieron más seguros y confiados para enfrentar la cirugía de emergencia de pacientes sospechosos o contaminados por el nuevo coronavirus.
\end{abstract}

Palabras clave: Personal de salud; Simulación; Seguridad del paciente; Infecciones por coronavirus.

\title{
1. Introdução
}

A Organização Mundial da Saúde (OMS) divulgou em dezembro de 2019 que as autoridades chinesas detectaram um novo vírus, identificado em uma pessoa hospitalizada com pneumonia em Wuhan, na China, posteriormente denominado SARS-CoV-2. Um ano após, o número de casos atingiu milhões de pessoas e o ataque viral ceifou milhares de vidas. O mundo vivencia uma pandemia sem precedentes na história contemporânea (Ribeiro, Oliveira, Silva, \& Souza, 2020).

Os profissionais de saúde estão diretamente implicados no atendimento às pessoas infectadas pela COVID-19 e, por esse motivo, compõem um grupo de risco específico para a infecção. A pandemia em curso expôs a fragilidade do setor de saúde em garantir a segurança dos profissionais envolvidos no cuidado aos infectados. Trata-se de uma exposição que pode ser compreendida como "exposição biológica" e a maioria, se não todos os profissionais de saúde, está exposta e possui alto risco de adquirir a doença, particularmente ao realizar procedimentos em vias aéreas ou próximos a elas (Ribeiro et al., 2020).

Segundo Adalja, Toner e Inglesby (2020), este sem dúvida, é um dos grandes desafios dessa pandemia: garantir a segurança e a proteção efetiva dos trabalhadores da saúde em um cenário de muitas dúvidas. A pandemia pelo SARS-CoV-2, agente da doença denominada COVID-19, gerou preocupação mundial, sobretudo aos sistemas de saúde, e considerando que os procedimentos cirúrgicos podem acelerar e agravar a progressão da COVID-19, situações em que a cirurgia não puder ser adiada é necessário garantir instalações adequadas para realizar o cuidado ideal com a máxima segurança (Agência Nacional de Vigilância Sanitária [ANVISA], 2020).

Gong et al. (2020), considera que no atual contexto da pandemia por SARS-CoV-2, um protocolo deve ser implementado para pacientes que necessitam de cirurgia, abordando preparação pré-operatória, o gerenciamento intraoperatório e a vigilância pós-operatória, com vistas a evitar complicações e garantir a segurança dos pacientes e da equipe de saúde. Nas cirurgias eletivas essenciais, como as oncológicas, devem-se avaliar os fatores de risco e benefício da realização do procedimento, assim como o momento ideal para a execução. Isso ocorre, pois pacientes com doenças malignas podem progredir ou apresentar sintomas que requeiram cuidados urgentes (ANVISA, 2020).

Todos os pacientes com suspeita de COVID-19 que necessitem de intervenção cirúrgica devem ser tratados como positivos até prova contrária, a fim de minimizar a propagação da infecção (Rocha et al., 2020). O centro cirúrgico é um cenário com uma dinâmica particular, onde a pressão pelo tempo, resultados, práticas complexas e multiprofissionais, marcam o cotidiano. Trata-se de um ambiente estressante e hostil devido à demanda de tarefas, o que estimula o silêncio e o distanciamento entre a equipe multiprofissional e o paciente, se transformando em um local com riscos elevados ao paciente (Ribeiro, Ferraz, \& Duran, 2017).

Barreto et al. (2020), considerou insuficiente o conhecimento sobre as formas de tratar e controlar a doença e, principalmente, a sua alta transmissibilidade e velocidade de disseminação. Esses riscos são uma realidade presente na 
assistência cirúrgica e cabe às equipes envolvidas no processo propor estratégias e estabelecer barreiras para garantir a segurança do paciente. Porém, a segurança não está limitada somente aos pacientes, mas aos profissionais de saúde que fazem parte de todo processo cirúrgico, como equipe de enfermagem; cirurgiões; anestesistas; patologistas; residentes de enfermagem, de cirurgia, de anestesia; profissionais de higienização, maqueiros e outros que possam estar envolvidos no processo.

Durante a pandemia, sentimentos de tristeza, medo e insegurança vieram à tona diante do risco de adoecimento dos profissionais de saúde pelo novo cenário que estava por vir de pacientes de emergência, suspeitos ou confirmados com COVID-19. A principal preocupação estava na contaminação desses profissionais, demonstrada pelos mesmos ao solicitarem a prática através da simulação realística, acreditando que os tornariam mais seguros.

Diante disso, emergiu da categoria de enfermagem, de uma unidade hospitalar de um Instituto de Oncologia do Rio de Janeiro, a sugestão de simular o cenário do atendimento a esse paciente, suspeito ou confirmado pelo novo coronavírus, a fim de reduzir ao máximo a condição de contaminação, visto que ainda existia o agravante de uma redução de aproximadamente $50 \%$ de profissionais de enfermagem em função de contaminação pelo SARS-CoV-2, assim como afastamentos por pertencerem ao grupo de risco. Recomenda-se que os profissionais selecionados para atendimento a esses pacientes não apresentem características definidas como grupo de risco (diabetes, hipertensão e doenças cardiovasculares) (Zheng et al., 2020).

Para os que permanecerem na linha de frente, resta vencer o medo, a ansiedade e a insegurança através de muito treinamento. É preciso assegurar que os profissionais que participarão desses atendimentos tenham o treinamento adequado sobre as técnicas de precaução padrão, por contato e por aerossóis (Wu et al., 2020). Utilizar Equipamento de Proteção Individual (EPI) adequado (touca, avental impermeável ou protetor de corpo plástico transparente sob capote de tecido de algodão, óculos ou protetor facial (Face Shield), respirador ou máscara N95, luvas que cubram o punho do avental, sapatos fechados e impermeáveis que permitam desinfecção). Higienizar as mãos antes e após colocar e retirar o EPI (ANVISA, 2020). Foi reforçada a norma regulamentadora (NR32), que define que é vedado nos serviços de saúde o uso de adornos (Brasil, 2005).

Não devem ser utilizadas alianças, anéis, pulseiras, relógio, colares, brincos, piercings expostos, toucas de tecido e crachás pendurados por cordão (Associação Brasileira de Enfermeiros de Centro Cirúrgico, Recuperação Anestésica e Centro de Material e Esterilização [SOBECC], 2020). O coronavírus sobrevive em diferentes superfícies, como por exemplo, plástico e aço inoxidável por 72 horas; cobre por menos de 4 horas e papelão por no máximo 24 horas (Adalja, Toner, \& Inglesby, 2020). Devido à sobrevida do vírus, o uso de adornos aumenta o risco de infecção. Foi nesse momento que evidenciou-se a necessidade de treinar a equipe através da metodologia ativa de simulação realística, indo de encontro ao conselho e orientação da própria Agência Nacional de Vigilância Sanitária (ANVISA) para estimular a realização de simulações de situações críticas como ferramenta facilitadora para o ensino-aprendizagem no treinamento, conforme Nota Técnica da ANVISA (ANVISA, 2020).

É importante a elaboração de protocolos e checklists específicos (recomenda-se, minimamente, o Protocolo de Cirurgia Segura - Lista de verificação de segurança cirúrgica e outro protocolo para paramentação e desparamentação de EPIs), com o objetivo de otimizar o fluxo de trabalho e prevenir infecções e outros eventos adversos (Organização Mundial da Saúde [OMS], 2009, Brasil, 2013).

Entre as estratégias da metodologia ativa de ensino, destaca-se a simulação realística, que, como estratégia do ensino em saúde, é definida como uma técnica que utiliza tecnologias para replicar cenários que simulam a prática em um ambiente controlado e realista. Nele, o aluno participa ativamente do processo de ensino-aprendizagem para praticar exaustivamente, 
aprender, refletir e avaliar produtos e processos. Ao realizar cenários, o aluno se depara com uma realidade que exigirá seu conhecimento para sua resolução (Janicas \& Narchi, 2019).

As etapas da simulação realística permitem discussões reflexivas (debriefing) sobre a situação ocorrida; sobre a aprendizagem e as decisões tomadas; estimula o pensamento crítico e reflexivo do estudante; e consolida os saberes. A simulação na educação dos profissionais de saúde dá aos participantes a oportunidade de desenvolverem habilidades variadas e competências necessárias em ambiente controlado e protegido, permite erros e crescimento profissional, sem arriscar a segurança do paciente (Ferreira, Guedes, Oliveira, \& Miranda, 2018).

A fim de evitar riscos e danos aos pacientes e aos profissionais de saúde, acredita-se que a simulação realística vem como metodologia ativa de ensino, oferecendo oportunidade de inserção em um ambiente muito próximo do real, de forma segura e controlada. Alves et al. (2019) concluem que a simulação realística se apresenta como uma estratégia eficaz de ensino e aprendizagem, pois permite a vivência prévia da prática da enfermagem, o que possibilita a reflexão acerca do papel do enfermeiro em diferentes contextos.

Perante o exposto, o objetivo deste artigo é descrever a prática e os resultados do uso da simulação realística in situ, a fim de simular o fluxo e atendimento do paciente cirúrgico com suspeita ou confirmação de contaminação do SARS-CoV-2 no período transoperatório.

\section{Metodologia}

\subsection{Tipo de estudo e período}

Trata-se de um estudo descritivo, com abordagem qualitativa, do tipo relato de experiência. As metodologias ativas para o ensino na área da saúde vêm crescendo cada vez mais, gerando qualidade e melhor curva de retenção de conhecimentos pós-aplicação. Uma das metodologias de ensino que parece vir garantindo uma prática consubstanciada na evolução do processo de ensino-aprendizagem é a Simulação Realística. Ela vem sendo utilizada como estratégia de ensino e vem sendo utilizada para a formação em enfermagem, principalmente para atividades práticas, na Simulação Realística, o discente é o sujeito ativo da aprendizagem, em cenários estratégicos e previamente criados, de modo a garantir a qualidade do ensino (Espadaro, 2019).

A simulação realística como prática de treinamento foi realizada no período de abril e maio do ano de 2020, logo após o período inicial da manifestação do SARS-CoV-2 no Brasil. Com a redução do mapa cirúrgico eletivo, houve uma otimização do tempo para a aplicação do treinamento, através da simulação realística, com os profissionais escalados do dia. O aprendizado baseado em simulação é uma metodologia eficaz para o desenvolvimento de conhecimentos, habilidades e atitudes, e ao mesmo tempo protegendo os pacientes de riscos desnecessários (Scalabrini et al., 2017).

\subsection{Cenário do estudo}

O cenário foi planejado reportando o mais próximo possível da realidade, ocorreu no próprio local (centro cirúrgico) da unidade hospitalar de um instituto de oncologia do Rio de Janeiro/RJ, onde foi criado o fluxo de atendimento para cirurgia de urgência/emergência, para realização da simulação realística. Foram montados cenários abrangendo todo o percurso do paciente, desde a entrada no corredor de acesso até à sua admissão dentro da própria sala de cirurgia, visto que eram pacientes de urgência/emergência (pacientes sem a possibilidade de teste para confirmação de infecção por coronavírus) e não poderiam passar pela sala de admissão; momento da intubação, transoperatório e saída desse paciente direto para leito de origem ou cti, conforme indicação médica. 


\subsection{Sujeitos envolvidos na experiência}

Os participantes da simulação realística foram os profissionais lotados no centro cirúrgico, de diferentes categorias. Para este estudo participaram 51 profissionais de saúde.

\subsection{Descrição da experiência}

As atividades se iniciaram com a formulação de protocolos de segurança dos pacientes e profissionais, definidos pela instituição através do Serviço de Controle de Infecção Hospitalar (SCIH), apresentados para as chefias dos serviços que integram o processo do centro cirúrgico com participação nas definições das especificidades de cada categoria. Essa etapa foi primordial para promover dados relevantes a respeito da temática e, consequentemente, instrumentalizar o facilitador na elaboração do cenário e a simulação realística.

Para que as práticas simuladas fossem efetivas, foi necessário planejar cada etapa obedecendo a um nível crescente de complexidade, o que possibilitou aos participantes demonstrarem competência adquirida em cada fase da simulação do processo.

Foi realizado o briefing com tempo de 15 minutos, com a finalidade de apresentar as situações-problemas, os objetivos de aprendizagem, além de todo o ambiente do cenário (centro cirúrgico in situ), equipamentos, insumos, mobiliários e ator (voluntário da equipe) fazendo papel de paciente padronizado, também orientações das etapas de todo o processo.

A simulação realística teve um tempo estabelecido de 25 a 30 minutos, onde foi considerada a ordem das etapas dos acontecimentos, iniciada pelo acolhimento do paciente no centro cirúrgico e encaminhamento direto para sala de cirurgia, onde somente o anestesista com o auxílio de um residente de anestesia ou técnico de enfermagem assistente da anestesia permaneciam na sala de cirurgia para intubação, um técnico de enfermagem com a função de circulante externo(corredor) permanecia na porta da sala assistindo momento da intubação pelo visor de vidro para ao término da mesma, sinalizar à equipe cirúrgica o momento do início da degermação das mãos, seguido do momento de se paramentar em uma sala específica montada para essa finalidade, aguardando a enfermeira responsável sinalizar o momento para entrada em bloco (todos juntos ao mesmo tempo) na sala cirúrgica com o propósito de reduzir a quantidade de vezes de abertura da porta.

Ao término do procedimento cirúrgico, todos os membros da equipe se desparamentavam na técnica preconizada pelo serviço de controle de infecção hospitalar-SCIH. Porém, o anestesista e enfermeiro responsáveis pelo retorno do paciente à unidade de enfermaria ou centro de tratamento intensivo-CTI conforme indicação médica, recebia do técnico do corredor outro capote para encaminhar o paciente, foi estabelecida uma sala de guarda dos equipamentos de proteção individual - EPIs, devidamente desinfetados e personalizados em armários específicos por categoria profissional para facilitar a retirada e guarda dos mesmos.

Utilizou-se a estratégia de simulação de alto rendimento através da Prática Deliberada de Ciclos Rápidos (PDCR) que tem como objetivo melhorar a performance dos participantes para alcançarem a maestria em uma habilidade, repetindo-se inúmeras vezes para memorização, sem constrangimento de cada participante, a fim de reduzir ao máximo o erro com foco no acerto (Castro \& Couto, 2018).

A comunicação entre os profissionais, durante todo o período do fluxo de entrada e saída do paciente e assistência ao processo cirúrgico, tinha seu papel importante durante a simulação realística, a fim de melhorar a interação desses profissionais e contribuir pela segurança do paciente.

Foi observado o erro mais frequente, o que motivava esse erro, a razão do erro ainda estar acontecendo e, ao final do treinamento, realizou-se o debriefing com duração de 40 minutos, para extrair contribuições dos participantes e reforçar os 
pontos positivos. O erro não foi valorizado, mas mostrou-se a solução com desfecho coletivo para implantação do protocolo de atendimento ao paciente suspeito ou confirmado para a COVID-19, trabalhou-se a monitoria do aprender para ensinar.

\section{Resultados e Discussão}

A iniciativa deu-se a partir da necessidade de aproximação com o novo, em vista a ansiedade e o medo que permeava os profissionais do centro cirúrgico para realizar o procedimento com paciente contaminado pelo novo coronavírus. Foi importante compreender o sentimento de ansiedade e medo que os profissionais envolvidos tinham sobre o assunto, e esse fator colaborou e influenciou para a estratégia metodológica.

Quanto aos participantes da simulação realística, foram 51 profissionais de categorias diferentes, como enfermeiros (04), técnicos de enfermagem (20), anestesistas (16), residentes do Programa de Residência Multiprofissional em Oncologia, sendo de enfermagem (12) e de anestesia (04), maqueiros (03) e profissionais de higienização (04) lotados no centro cirúrgico da unidade hospitalar $\mathrm{n}^{\circ} 2$ de um instituto de oncologia do Rio de Janeiro/RJ, alguns desses profissionais atuaram fazendo o papel do paciente padronizado.

O fato de o cenário ter sido estruturado no próprio local de trabalho facilitou bastante o entendimento de todo o fluxo e processo de atendimento ao paciente suspeito ou contaminado pelo SARS-CoV-2. O protocolo foi construído a partir das evidências e revisado frequentemente no avançar da pandemia, e essa construção tornou-se muito significativa, por nortear a conduta dos profissionais.

Foram descritas as atribuições das equipes e os procedimentos inerentes ao setor do centro cirúrgico. Não apenas de cunho individual, mas também de responsabilidade coletiva, sendo necessário reconhecer as potencialidades e fragilidades dentro do processo. Um dos maiores desafios, se não o maior, foi manter-se atualizado em meio a tanto dinamismo.

As atividades de simulação devem considerar toda complexidade que as envolve e, com isso, ser possível identificar as competências a serem desenvolvidas pelo participante em cada fase dessa estratégia de aprendizagem significativa (Fabri et al., 2017). Por meio dos objetivos traçados através da simulação realística, o facilitador definiu os conteúdos abordados, as intervenções esperadas e as competências a serem adquiridas pelos aprendizes na execução do cenário.

Após refletir sobre as situações-problemas apresentadas, construiu-se os objetivos de aprendizagem e definiu-se pela estratégia da simulação realística como estratégia de treinamento, conforme Quadro 1.

Quadro 1. Objetivos de aprendizagem, situação-problema, público-alvo e estratégias de simulação.

\begin{tabular}{|l|l|l|l|}
\hline Objetivos de aprendizagem & Situação problema & Público-alvo & Estratégias de simulação \\
\hline $\begin{array}{l}\text { Desenvolver habilidade, no fluxo e } \\
\text { processo de atendimento do paciente } \\
\text { cirúrgico suspeito ou contaminado } \\
\text { pelo SARS-CoV-2 no período } \\
\text { transoperatório. }\end{array}$ & $\begin{array}{l}\text { Assegurar a confiança, segurança dos } \\
\text { profissionais do centro cirúrgico na } \\
\text { assistência ao paciente suspeito ou } \\
\text { contaminado pelo SARS-CoV-2. }\end{array}$ & $\begin{array}{l}\text { Enfermeiros; técnicos de } \\
\text { enfermagem; anestesistas; } \\
\text { residentes de anestesia e } \\
\text { enfermagem; maqueiros e } \\
\text { profissionais de higienização. }\end{array}$ & $\begin{array}{l}\text { Simulação realística in situ } \\
\text { com paciente padronizado. }\end{array}$ \\
\hline $\begin{array}{l}\text { Treinar o protocolo (SCIH) de } \\
\text { técnicas de precaução padrão, por } \\
\text { contato e por aerossois, } \\
\text { paramentação/desparamentação para } \\
\text { assistência ao paciente cirúrgico } \\
\text { suspeito ou contaminado pelo SARS- } \\
\text { CoV-2 no período transoperatório. }\end{array}$ & $\begin{array}{l}\text { Evitar contaminação durante } \\
\text { assistência ao paciente cirúrgico } \\
\text { suspeito ou contaminado pelo SARS- } \\
\text { CoV-2; técnica de } \\
\text { paramentação/desparamentação. }\end{array}$ & $\begin{array}{l}\text { Enfermeiros; técnicos de } \\
\text { enfermagem; anestesistas; } \\
\text { residentes de anestesia e } \\
\text { enfermagem; maqueiros e } \\
\text { profissionais de higienização. }\end{array}$ & $\begin{array}{l}\text { Práticas Deliberadas de } \\
\text { ciclos rápidos - PDCR }\end{array}$ \\
\hline $\begin{array}{l}\text { Aperfeiçoar as habilidades da } \\
\text { comunicação entre os profissionais } \\
\text { durante todo o processo no período } \\
\text { transoperatório }\end{array}$ & $\begin{array}{l}\text { Melhorar interação entre os } \\
\text { profissionais do centro cirúrgico } \\
\text { durante o atendimento ao paciente } \\
\text { cirúrgico suspeito ou contaminado } \\
\text { pelo SARS-CoV-2 no período } \\
\text { transoperatório. }\end{array}$ & $\begin{array}{l}\text { Enfermeiros; técnicos de } \\
\text { enfermagem; anestesistas; } \\
\text { residentes de anestesia e } \\
\text { enfermagem; maqueiros e } \\
\text { profissionais de higienização. }\end{array}$ & $\begin{array}{l}\text { Simulação realística in } \\
\text { situ. }\end{array}$ \\
\hline
\end{tabular}


Quanto à veracidade da situação do cenário, buscou-se a imitar uma situação real, a utilização do cenário in situ foi um facilitador ao processo, deixando os participantes mais à vontade em desenvolver a simulação realística.

Nesse sentido, foi montado um protocolo do fluxo do paciente de emergência suspeito ou confirmado de contaminação pelo SARS-CoV-2, abrangendo as seguintes fases: trajeto de saída do leito de origem (unidade de pronto atendimento, enfermaria ou terapia intensiva); preparo da sala com mínimo de material necessário; momento da intubação; preparo de uma sala para a equipe cirúrgica se paramentar fora da sala de cirurgia e entrada, de todos juntos, em bloco para sala operatória; circulante externo atendendo pelo vidro as solicitações por escrito do circulante interno de forma legível e aumentada; desparamentação em sala e local próprio designado para guarda das Faces Shields individuais, para evitar a contaminação domiciliar; e saída desse paciente para leito de origem com a presença do anestesista, enfermeiro e maqueiro devidamente paramentado. No Quadro 2 está exemplificado as etapas do cenário da simulação realística.

Quadro 2. Descrição do cenário sobre fluxo e atendimento do paciente cirúrgico com suspeita ou confirmação de contaminação do SARS-CoV-2 no período transoperatório.

\begin{tabular}{|c|c|}
\hline Tema proposto & $\begin{array}{l}\text { Fluxo e atendimento do paciente cirúrgico com suspeita ou confirmação de } \\
\text { contaminação do SARS-CoV-2 no período transoperatório. }\end{array}$ \\
\hline Público-alvo & $\begin{array}{l}\text { Enfermeiros, técnicos de enfermagem, anestesistas, residentes de enfermagem e } \\
\text { anestesia do Programa de Residência Multiprofissional em Oncologia, maqueiros e } \\
\text { profissionais de higienização. }\end{array}$ \\
\hline Conhecimento prévio do participante & $\begin{array}{l}\text { Protocolos determinados pela Instituição hospitalar através do Serviço de controle } \\
\text { de infecção hospitalar, divisão de enfermagem e coordenação do centro cirúrgico, } \\
\text { demonstrados aos profissionais por vídeos aulas e presencialmente. }\end{array}$ \\
\hline Modalidade de Simulação & $\begin{array}{l}\text { Simulação clínica com uso de paciente padronizado; Prática deliberada de ciclos } \\
\text { rápidos-PDCR; Simulação in situ. }\end{array}$ \\
\hline $\begin{array}{l}\text { Recursos humanos para condução do } \\
\text { cenário }\end{array}$ & $\begin{array}{l}\text { Paciente padronizado; profissionais padronizados; facilitador do cenário e } \\
\text { debriefing. }\end{array}$ \\
\hline Recursos materiais & $\begin{array}{l}\text { Maca de transporte, travesseiro, lençol, pulseira de identificação (descritos nome } \\
\text { completo, matrícula hospitalar, data nascimento), prontuário simulado, luva de } \\
\text { procedimento, frasco de álcool 70\%, óculos de proteção, máscaras PFF@ (N95), } \\
\text { Protetor de face (face shield), capote de proteção, lixeiras (infectante e comum), } \\
\text { caixa de pérfuro-cortante, prancheta, caneta, mesa cirúrgica, mesa auxiliar, materiais } \\
\text { de intubação, dispensador de álcool gel, carro de medicamentos. }\end{array}$ \\
\hline Equipamentos & Não utilizado. \\
\hline Descrição do ambiente & $\begin{array}{l}\text { Ambiente do centro cirúrgico incluindo: corredor, recuo do lavabo, sala de cirurgia } \\
\text { determinada e reservada para procedimentos cirúrgicos de pacientes suspeitos ou } \\
\text { contaminados pelo Sars Cov-2, sala de guarda de EPIs e para paramentação. } \\
\text { Paciente padronizado: vestido de camisola hospitalar e pulseira de identificação. } \\
\text { Documentação: Prontuário. }\end{array}$ \\
\hline Caso clínico & $\begin{array}{l}\text { Paciente M. C. A, } 42 \text { anos, proveniente da unidade de emergência, onde foi } \\
\text { realizado coleta de swab nasal (PCR) para infecção pelo Sars Cov-2, sem tempo } \\
\text { hábil para resultado, tornando-a suspeita ou contaminado pelo coronavírus, foi } \\
\text { admitida no centro cirúrgico para laparotomia exploradora por obstrução intestinal } \\
\text { proveniente de carcinoma de ovário aderido ao sigmoide. }\end{array}$ \\
\hline Informações para os participantes & $\begin{array}{l}\text { Realizar todas as etapas de admissão e encaminhamento do paciente a sala de } \\
\text { cirurgia, utilização dos EPIs, paramentação/desparamentação, intubação, } \\
\text { procedimento cirúrgico, retorno do paciente ao leito determinado. }\end{array}$ \\
\hline
\end{tabular}

Fonte: Autoras.

Todos os profissionais de saúde têm a responsabilidade de fazer a gestão de risco observando as ações de melhorias que modificam os processos, as rotinas assistenciais e as práticas. Em decorrência disso, melhora o nível de segurança para evitar os eventos adversos, os danos aos pacientes. 
É incontestável admitir que foi proativa a manifestação de desejo da equipe em simular o cenário da nova situação de pacientes suspeitos ou confirmados por infecção do Sars Cov-2, para contribuir como uma barreira garantindo o nível de segurança na assistência prestada a esse paciente cirúrgico.

Quanto à ferramenta de educação em saúde utilizada, destacou-se na fala dos profissionais que a simulação realística consistiu em uma escolha perfeita, onde possibilitou o desenvolvimento de aprendizagens de fluxos e procedimentos ligados ao momento da pandemia no setor de centro cirúrgico. Além disso, as habilidades técnicas e comportamentais puderam ser melhoradas, contribuindo no processo decisório.

Observou-se também que o relacionamento entre as equipes para a prática assistencial foi aperfeiçoado após a realização da ferramenta metodológica. Maior iniciativa de decisões no trabalho e maior motivação para a adesão de protocolos locais são desenvolvidas por profissionais que participam de oficinas (Santos, Pedrosa, \& Pinto, 2016).

Não houve pré e pós-teste à simulação realística, porém o que motivou foi a solicitação dos profissionais a simularmos o período transoperatório de atendimento ao paciente suspeito ou contaminado por infecção do Sars Cov-2 e após a estratégia de treinamento, tivemos como resultado algumas falas registradas dos profissionais que participaram da simulação:

\footnotetext{
“Como foi bom simularmos...”.

"Agora me sinto bem mais segura...".

"Estamos mais preparadas para o enfrentamento...".

“Agora vai...".

"Juntos somos mais fortes...".

"Podíamos sempre fazer simulação...".

"Simulação é uma excelente ferramenta pra treinar...".

"Continuamos preocupados com o novo, porém mais seguros...".
}

\section{Considerações Finais}

Considera-se que a busca, por parte da equipe, de evidências que surgiram, bem como de estratégias desenvolvidas para aplicação e adequação das recomendações publicadas pelos institutos, associações e órgãos governamentais, possibilitou a melhora do desempenho da equipe em relação à assistência ao paciente suspeito ou contaminado pelo SARS-CoV-2, visando à segurança dos profissionais e dos pacientes. O desenvolvimento coletivo do saber-fazer tornou todos os envolvidos mais seguros e conhecedores do seu processo de trabalho.

Vale ressaltar que o protocolo foi construído e implementado no primeiro momento da contaminação da pandemia de COVID-19, onde tudo era muito novo em todo o mundo e grande parte dos profissionais do setor foi afastada devido à contaminação pelo novo coronavírus e por pertencerem à grupos de risco. Entende-se que o protocolo instituído através da simulação realística conferiu segurança à equipe para execução da assistência ao paciente com suspeita ou infecção confirmada de COVID-19.

Este estudo permite, assim, evidenciar a importância da simulação realística no contexto hospitalar, uma vez que esse é um dos métodos mais eficazes de ensino-aprendizagem disponíveis atualmente. Ademais, cabe mencionar que o seu uso no ensino na área da saúde é de grande valia, já que pode ser utilizada como método de aprendizagem, aperfeiçoamento e atualização em diversos contextos. 
Em ordem decrescente, destacam-se aqui as palavras que resumem os sentimentos dos profissionais após treinamento facilitado pela já relatada simulação realística: segurança, confiança, tranquilidade, redução do medo, firmeza, melhor assistência e capacidade.

Em se tratando da especificidade de oncologia, o tempo é crucial. As cirurgias por traumas reduziram significativamente pelo recolhimento das pessoas às ruas, mas as células cancerosas não entenderam esse momento e persistiram em se multiplicar. Então, restou aos profissionais da linha de frente da cirurgia abraçar a causa da prática educativa, através da simulação realística, para vencer o medo e a ansiedade e conquistar a confiança, sendo o objetivo principal da metodologia ativa.

\section{Referências}

Adalja, A. A., Toner, E., \& Inglesby, T. V. (2020). Priorities for the US health community responding to COVID-19. JAMA, 323(14), $1343-1344$.

Agência Nacional de Vigilância Sanitária - ANVISA (2020). Orientações para serviços de saúde: medidas de prevenção e controle que devem ser adotadas durante a assistência aos casos suspeitos ou confirmados de infecção pelo novo coronavírus (SARS-CoV-2). ANVISA. https://portaldeboaspraticas.iff.fiocruz.br/wp-content/uploads/2021/03/NOTA-TECNICA-GVIMS_GGTES_ANVISA-04_2020-25.02-para-o-site-1.pdf

Alves, N. P., Gomes, T. G., Lopes, M. M. C. O., Gubert, F. A., Lima, M. A., Beserra, E. P., \& Cavalcante, V. M. V. (2019). Simulação realística para a formação do enfermeiro. Revista de Enfermagem UFPE On Line, 13(5), 1420-1428.

Associação Brasileira de Enfermeiros de Centro Cirúrgico, Recuperação Anestésica e Centro de Material e Esterilização - SOBECC (2020). Recomendações relacionadas ao fluxo de atendimento para pacientes com suspeita ou infecção confirmada pelo COVID-19 em procedimentos cirúrgicos ou endoscópicos. Cerqueira César, SP: SOBECC. http://www.coren-es.org.br/wp-content/uploads/2020/04/RECOMENDA\%C3\%87\%C3\%95ES-_SOBECC_COVID_2\%C2 $\%$ BA-EDI\%C3\%87\%C3\%83O-1.pdf

Barreto, M. L., Barros, A. J. D., Carvalho, M. S., Codeço, C. T., Hallal, P. R. C., Medronho, R. A., \& Werneck, G. L. (2020). O que é urgente e necessário para subsidiar as políticas de enfrentamento da pandemia de COVID-19 no Brasil? Revista Brasileira de Epidemiologia, 23, e200032.

Brasil (2005). Portaria MTE n. 485, de 11 de novembro de 2005. Estabelece as diretrizes básicas para a implementação de medidas de proteção à segurança $e$ à saúde dos trabalhadores dos serviços de saúde, bem como daqueles que exercem atividades de promoção e assistência à saúde em geral. https://www.saude.mg.gov.br/images/documentos/Portaria_485.pdf

Brasil (2013). Portaria n. 2.095, de 24 de setembro de 2013. Aprova os Protocolos Básicos de Segurança do Paciente. https://www.saude.pr.gov.br/sites/default/arquivos_restritos/files/documento/2020-05/portaria_2095_2013.pdf

Castro, L. D., \& Couto, T. B. (2018). Rapid Cycle Deliberate Practice: a modern simulation strategy. Scientia Medica, 28(1), ID28849.

Espadaro, R. F. Simulação realística na formação em enfermagem. 2017. 116 f. Dissertação (Programa de Pós-Graduação em Educação) - Universidade Nove de Julho, São Paulo, 2017.

Fabri, R. P., Mazzo, A., Martins, J. C. A., Fonseca, A. S., Pedersoli, C. E., Miranda, F. B. G., \& Baptista, R. C. N. (2017). Construção de um roteiro teóricoprático para simulação clínica. Revista da Escola de Enfermagem da USP, 51, e03218.

Ferreira, R. P. N., Guedes, H. M., Oliveira, D. W. D., \& Miranda, J. L. (2018). Simulação Realística como Estratégia de Ensino no Aprendizado de Estudantes da Área da Saúde. Revista de Enfermagem do Centro-Oeste Mineiro, 8, e2508.

Gong, Y., Cao, X., Mei, W., Wang, J., Shen, L., Wang, S., \& Huang, Y. (2020). Anesthesia Considerations and Infection Precautions for Trauma and Acute Care Cases During the COVID-19 Pandemic: Recommendations From a Task Force of the Chinese Society of Anesthesiology. Anesthesia \& Analgesia, 131(2), 326-334.

Janicas, R. C. S. V., \& Narchi, N. Z. (2019). Evaluation of nursing students' learning using realistic scenarios with and without debriefing. Revista LatinoAmericana de Enfermagem, 27, e3187.

Organização Mundial da Saúde - OMS (2009). Segundo desafio global para a segurança do paciente: cirurgias seguram salvam vidas (orientações para cirurgia segura da OMS). OMS. http://bvsms.saude.gov.br/bvs/publicacoes/seguranca_paciente_cirurgias_seguras_salvam_vidas.pdf

Ribeiro, A. P., Oliveira, G. L., Silva, L. S., \& Souza, E. R. (2020). Saúde e Segurança de Profissionais de Saúde no Atendimento a Pacientes no Contexto da Pandemia de Covid-19: Revisão de Literatura. Revista Brasileira de Saúde Ocupacional, 45, e25.

Ribeiro, E., Ferraz, K. M. C., \& Duran, E. C. M. (2017). Atitudes dos enfermeiros de centro cirúrgico diante da Sistematização da Assistência de Enfermagem Perioperatória. Revista SOBECC, 22(4), 201-207.

Rocha, L. P., Castanheira, J. S., Barlem, E. L. D., Carvalho, D. P., Gutierres, E. D., Passos, C. M., \& Brum, R. G. (2020). Paciente cirúrgico no contexto da pandemia de COVID- 19. Escola de Enfermagem da Universidade Federal do Rio Grande. https://eenf.furg.br/images/COVID/Paciente_Cirurgico_n o_Contexto_da_Pandemia_de_COVID-19.pdf 
Research, Society and Development, v. 10, n. 10, e451101019017, 2021

(CC BY 4.0) | ISSN 2525-3409 | DOI: http://dx.doi.org/10.33448/rsd-v10i10.19017

Santos, P. F., Pedrosa, K. A., Pinto, J. R. (2016). A Educação Permanente como ferramenta no trabalho interprofissional na Atenção Primária à Saúde. Tempus - Actas de Saúde Coletiva, 10(3), 177-189.

Scalabrini, A., Fonseca, A., \& Brandão, C. F. S. Simulação realística e habilidades na saúde. Atheneu, 2017.

Wu, F., Zhao, S., Yu, B., Chen, Y., Wang, W., Song, Z., \& Zhang, Y. (2020). A new coronavirus associated with human respiratory disease in China. Nature, $579,265-269$.

Zheng, H., Hébert, H. L., Chatziperi, A., Meng, W., Smith, B. H., Yan, J., \& Colvin, L. A. (2020). Perioperative management of suspected or confirmed cases of COVID-19: review and recommendations for perioperative management from a retrospective cohort study. British Journal of Anaesthesia, 125(6), 895-911. 